\title{
Genetic testing of complement and coagulation pathways in patients with severe hypertension and renal microangiopathy
}

\author{
Christopher P Larsen, Jon D Wilson, Alejandro Best-Rocha, Marjorie L Beggs and \\ Randolph A Hennigar
}

Arkana Laboratories, Little Rock, AR, USA

\begin{abstract}
A diagnosis of thrombotic microangiopathy on kidney biopsy in a patient presenting with hypertensive emergency has historically elicited the diagnosis of malignant hypertension-associated thrombotic microangiopathy. Recent studies, however, have raised awareness that a number of these patients may actually represent atypical hemolytic uremic syndrome. To further investigate this premise, we performed next-generation sequencing to interrogate the coding regions of 29 complement and coagulation cascade genes associated with atypical hemolytic uremic syndrome in 100 non-elderly patients presenting with severe hypertension, renal failure and a kidney biopsy showing microangiopathic changes limited to the classic accelerated hypertensionassociated lesion of arterial intimal edema ('mucoid intimal hyperplasia') in isolation and without accompanying glomerular microthrombi. No pathogenic or likely pathogenic variants were identified in any of the genes analyzed, although 13 patients had rare variants of uncertain significance predicted to be deleterious by all insilico prediction methods utilized. Accordingly, this large patient cohort showed no definitive burden of disease secondary to genetic variants involving complement or coagulation pathways, which contrasts sharply with the high frequency of similar mutational events reported for atypical hemolytic uremic syndrome. Our results also inform recent data by suggesting that patients who present with severe or malignant hypertension and renal thrombotic microangiopathy may be at higher risk for atypical hemolytic uremic syndrome only if the biopsy shows more active disease that includes glomerular fibrin thrombi.

Modern Pathology (2018) 31, 488-494; doi:10.1038/modpathol.2017.154; published online 17 November 2017
\end{abstract}

The term 'thrombotic microangiopathy' is used to describe a broad category of acquired and hereditary diseases that are unified by the shared feature of microvascular endothelial injury. The clinical hallmarks include microangiopathic hemolytic anemia (ie, schistocytes on peripheral smear), thrombocytopenia and end-organ damage. Conditions that fall under the rubric of thrombotic microangiopathy include hemolytic uremic syndrome, thrombotic thrombocytopenia, autoimmune disease (systemic lupus erythematosus, antiphospholipid antibody syndrome, scleroderma), hypertensive emergency (accelerated and malignant hypertension), bacterial infections, viral infection (eg, HIV), pre-eclampsia, eclampsia, pregnancy-associated hemolysis elevated liver enzymes low platelets (HELLP), disseminated intravascular coagulation, pancreatitis, drugs (cancer

Correspondence: Dr CP Larsen, MD, Pathology, Arkana Laboratories, 10810 Executive Center Drive, Suite 100, Little Rock, AR 72211, USA.

E-mail: Chris.larsen@nephropath.com

Received 18 August 2017; revised 14 September 2017; accepted 15 September 2017; published online 17 November 2017 chemotherapeutics, interferon infusion, oral contraceptives, calcineurin inhibitors, quinine, cocaine, IV Opana, heroin), systemic cancer, coagulopathic disorders, acute radiation nephritis, post-surgical microangiopathic hemolytic anemia and thrombocytopenia, cobalamin C disease, heparin-induced thrombocytopenia and atypical hemolytic uremic syndrome.

Some of these disorders are linked to activation of complement pathways, with atypical hemolytic uremic syndrome as a prime example. About 60$70 \%$ of atypical hemolytic uremic syndrome patients harbor mutations affecting one or more genes encoding effector or regulatory elements of complement or coagulation pathways. ${ }^{1}$ Atypical hemolytic uremic syndrome can arise spontaneously or be triggered by certain conditions in patients who are genetically susceptible. These conditions include malignancy, autoimmune disease, viral and bacterial infections, drugs, pregnancy-related conditions, transplant rejection and malignant hypertension., ${ }^{2,3}$ Complement is often activated in these disorders and can spiral out of control in patients who lack the intrinsic ability to regulate it. Hence, this 
'unmasking' of atypical hemolytic uremic syndrome essentially represents a two-hit process.

Thrombotic microangiopathy in the setting of malignant hypertension is usually caused by mechanical stress resulting in platelet activation and thrombosis. However, some studies report patients presenting initially with severe hypertension, but subsequently showing features of atypical hemolytic uremic syndrome on further work-up. ${ }^{4-6}$ For example, Totina et $a 1^{5}$ described a 10 -month-old child who presented with hypertensive emergency and was later discovered to have atypical hemolytic uremic syndrome. A kidney biopsy was performed and subsequently confirmed active thrombotic microangiopathy. In this case, the absence of thrombocytopenia and renal injury at presentation did not prompt immediate consideration of atypical hemolytic uremic syndrome. Unfortunately, the child succumbed to her disease despite daily plasmapheresis with fresh-frozen plasma infusions. It may be argued that the vast majority of patients who present with severe hypertension and renal failure are not biopsied, as hypertensive nephrosclerosis is clinically inferred.

The article by Timmermans et $a l^{4}$ is especially noteworthy in documenting specific genetic defects of complement components and serologic evidence of complement activation in a small cohort of patients presenting with the triad of severe hypertension, renal failure and thrombotic microangiopathy on kidney biopsy. The biopsies showed active thrombotic microangiopathy lesions including mesangiolysis, fibrinoid arteritis, glomerular microthrombi, mucoid intimal edema and ischemic glomerular tuft collapse. The majority of patients were found to have genetic complement abnormalities commonly found in atypical hemolytic uremic syndrome. This report and others imply that patients who present similarly should be screened for complement abnormalities and treated accordingly. ${ }^{4-6}$ However, kidney biopsies from patients who present with the triad often do not exhibit the degree of morphologic activity reported by Timmermans and colleagues. Namely, arterial intimal edema or 'mucoid intimal hyperplasia' in isolation without accompanying glomerular fibrin thrombi has long been thought to be characteristic of malignant hypertension-associated thrombotic microangiopathy based on historical data. ${ }^{7,8}$ This practice is still common today as evidenced from recent case reports ${ }^{9,10}$ and the fact that two of the most recently published and widely circulated renal pathology textbooks both show photomicrographs of this lesion with a caption associating it with malignant hypertension-related thrombotic microangiopathy. ${ }^{11,12}$

Our study attempts to recapitulate in part the findings of Timmermans et al, but in patients whose kidney biopsies show activity limited to mucoid intimal edema of the arterial tree. We utilized nextgeneration sequencing technology to determine how often non-elderly patients with severe hypertension, renal failure and mucoid intimal edema display genetic variants in complement and/or coagulation pathway genes. Genes associated with focal segmental glomerulosclerosis and Alport's disease were also interrogated.

\section{Materials and methods}

We identified 100 consecutive kidney biopsies from non-elderly patients who exhibited severe hypertension, renal failure and morphologic findings consistent with thrombotic microangiopathy, which also had adequate DNA for sequencing. Patients were included in the study if (1) they were $<50$ years of age; (2) their blood pressure exceeded 180$/ 120 \mathrm{~mm} \mathrm{Hg}$ and was documented before biopsy; (3) a history of renal failure was reported before the biopsy; and (4) their biopsies showed arterial intimal
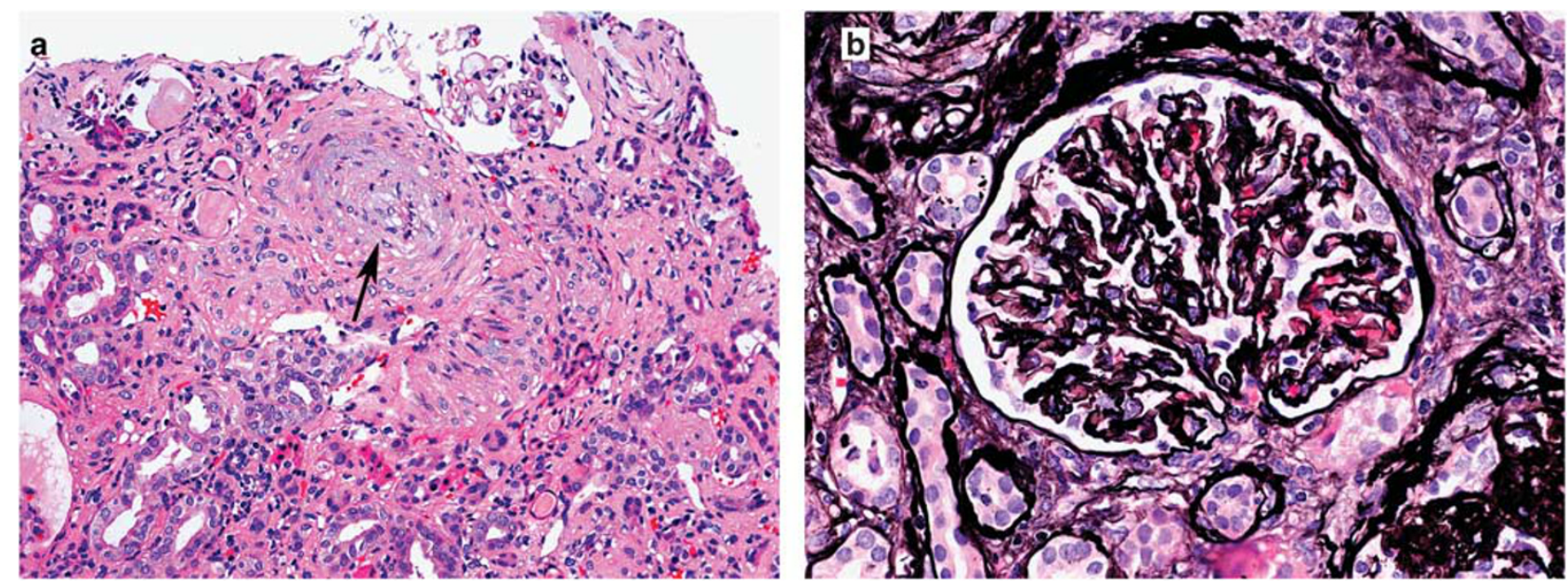

Figure 1 Hypertension-associated thrombotic microangiopathy. A renal biopsy from a patient with malignant hypertension shows (a) an artery occluded by intimal edema (hematoxylin and eosin; original magnification $\times 100)$ and $(\mathbf{b})$ a glomerulus with ischemic basement membrane wrinkling (Jones methenamine silver original magnification $\times 400$ ). 
edema or 'mucoid intimal hyperplasia' in isolation without accompanying glomerular microthrombi, mesangiolysis or necrotizing arteritis/arteriolitis (Figure 1). Cases with morphologic findings of thrombotic microangiopathy on kidney biopsy were excluded if they had a known secondary etiology such as malignancy, autoimmune disease, infection, drugs, pregnancy-related complications, etc. We also excluded cases with concurrent glomerulonephritis such as IgA nephropathy. The study protocol was approved by Solutions IRB (Little Rock, AR, USA) and conformed to the principles outlined in the Declaration of Helsinki.

Genomic DNA was extracted from the deidentified frozen kidney biopsy tissue using the Qiagen DNeasy Blood and Tissue Kit. The DNA concentration of each sample was determined using a Qubit 3 Fluorometer and the dsDNA HS kit (Invitrogen, Thermo Scientific, Wilmington, DE, USA). We then performed targeted genomic enrichment using a custom next-generation sequencing gene panel (Agilent Technologies, Santa Clara, CA, USA) with bait sequences to coding regions of genes associated with atypical hemolytic uremic syndrome, as well as those described in other forms of thrombotic microangiopathy and complement abnormalities. Paired end sequencing was performed on the MiSeq system using the PE v3 150 cycle kit. A batch size of eight samples was selected in order to provide the optimum depth of coverage across the target region as previously described. ${ }^{13}$ Specifically, the coding and splice site regions for the following genes were sequenced by next-generation sequencing: CFH, CFI, CD46 (MCP), CFB, CFHR5, C3, THBD, ADAMTS13, PLG, DGKE, CFHR1, CFHR2, CFHR3, CFHR4, C1QA, C1QB, C1QC, C2, C8A, C9, CR2, MASP1, MASP2, C1S, F12, FCN1, MMACHC, $P L G$ and $V W F$.

The FASTQ files generated by the MiSeq were streamed to Illumina BaseSpace where the data was assembled with the BWA Genome Alignment Software and the variants called by GATK Variant Caller. This produced a Variant Call Format (.VCF) file, which can be imported into Illumina Variant Interpreter. The FASTQ files were also imported into DNASTAR's SeqMan NGen (SM NGen) assembler and the data interrogated in DNASTAR ArrayStar and SeqMan Pro. We filtered for coding variants with a minor allele frequency $<1 \%$ and also manually looked for coding variants in APOL1. Variants were considered disease causing under strict criteria in accordance with the recently published ACMG guidelines for the interpretation of sequence variants. ${ }^{14}$ The variants classified as variants of uncertain significance were further divided according to the results of three in-silico software prediction methods (Mutation Taster, PolyPhen2 and SIFT) as to whether or not they were predicted to be pathogenic by all three methods.

\section{Results}

The patients included in this study had a mean age of 35.4 years and included 71 males and 29 females. There were 63 African-Americans, 28 non-Hispanic Caucasian, 4 Hispanic Caucasians, 4 Asians and 1 Native American. The patients had a mean serum $\mathrm{Cr}$ of $7.8 \mathrm{mg} / \mathrm{dl}$ (range $1.9-21.7 \mathrm{mg} / \mathrm{dl}$ ). The platelet count was known in 58 patients and decreased below 150000 in 33 (57\%). The kidney biopsies had evidence of arterial mucoid intimal edema without glomerular fibrin thrombi by light microscopy and a negative immunofluorescence evaluation, as per inclusion criteria. Therefore, glomeruli did not show evidence of immune complex deposition in any cases. Specifically, there were no cases with evidence of C3 glomerulopathy. Most cases (92\%) had evidence of background arteriosclerosis, which was scored as mild in $9 \%$, moderate in $16 \%$ and severe in $67 \%$. The kidney biopsies from these patients had a mean of 37.1 glomeruli with a mean of 9.7 being globally sclerotic $(26 \%)$. The degree of interstitial fibrosis was scored as severe in $43 \%$, moderate in $37 \%$, mild in $19 \%$ and none in $1 \%$. A total of 35 cases had subendothelial electron-lucent expansion along basement membranes by electron microscopic examination (Figure 2).

One patient had a heterozygous nonsynonymous c.966G > A SNP in DGKE (rs138924661), which is unlikely to be the etiology of disease in this patient as it was only present in the heterozygous state. There were a total of 49 variants in 40 patients that were determined to be of undetermined significance. Of these, 15 variants in 13 patients were predicted to be significant (damaging, deleterious or disease causing) by in-silico analysis (Table 1). The remaining 34 variants were either predicted to be benign by

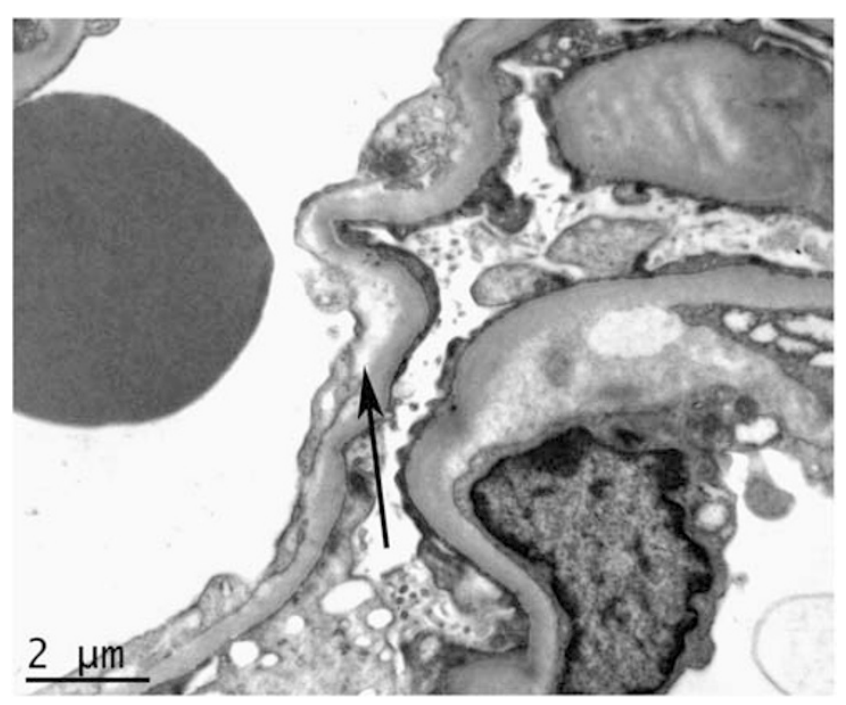

Figure 2 Electron microscopy showing glomerular basement membrane subendothelial electron-lucent expansion (arrow) from a biopsy of a patient with hypertension-associated thrombotic microangiopathy. 
all prediction methods (13 variants) or had mixed results (21 variants) (Table 2). There were 11 rare variants identified which were classified as 'likely benign'. A total of 52 patients had no rare variants identified in the complement and coagulation genes sequenced.
Genes associated with focal segmental glomerulosclerosis and Alport disease were also interrogated as a secondary analysis. There were no patients with known pathogenic mutations in any of these genes (ACTN4, ADCK3, ADCK4, ANLN, APOL1, APRT, ARHGAP24, ARHGDIA, CD2AP, CLCN5, COL4A1,

Table 1 Variants of uncertain significance in complement and coagulation genes predicted to be deleterious by all prediction methods

\begin{tabular}{|c|c|c|c|c|}
\hline Gene & Patient & AA change & Nucleotide & $d b S N P I D$ \\
\hline C2 & 1 & p.A472T & c. $1414 \mathrm{G}>\mathrm{A}$ & rs142243595 \\
\hline C9 & 2 & p.P167S & c. $499 \mathrm{C}>\mathrm{T}$ & rs34882957 \\
\hline C9 & 3 & p.P167S & c. $499 \mathrm{C}>\mathrm{T}$ & rs34882957 \\
\hline CFHR3 & 4 & Splice donor & c. $796+1 \mathrm{G}>\mathrm{A}$ & rs370108606 \\
\hline FCN1 & 5 & p.N289fs & c.866 delA & None \\
\hline$M A S P 1$ & 6 & p.G476R & c. $1426 \mathrm{G}>\mathrm{A}$ & rs140743904 \\
\hline MASP1 & 6 & p.G437R & c.1309G > A & rs147289826 \\
\hline MASP1 & 7 & p.R441fs & с.1322 1331delGCTCCCTGCC & None \\
\hline$M A S P 1$ & 8 & p.G437R & c. $1309 \mathrm{G}>\mathrm{A}$ & rs147289826 \\
\hline MASP2 & 9 & p.T128M & c. $383 \mathrm{C}>\mathrm{T}$ & rs141145402 \\
\hline MASP2 & 1 & p.T128M & c. $383 \mathrm{C}>\mathrm{T}$ & rs141145402 \\
\hline MASP2 & 10 & p.R356W & c. $1066 \mathrm{C}>\mathrm{T}$ & rs201990552 \\
\hline$P L G$ & 11 & p.T200A & c. $598 \mathrm{~A}>\mathrm{G}$ & rs149145958 \\
\hline PLG & 12 & p.R343W & c. $1027 \mathrm{C}>\mathrm{T}$ & rs142647215 \\
\hline$P L G$ & 13 & p.T200A & c. $598 \mathrm{~A}>\mathrm{G}$ & rs149145958 \\
\hline
\end{tabular}

All variants are heterozygous.

Table 2 Variants of uncertain significance in complement and coagulation genes predicted to not be deleterious by some or all prediction methods

\begin{tabular}{|c|c|c|c|c|c|}
\hline Gene & Patient & AA change & Nucleotide & $d b S N P I D$ & In-silico prediction \\
\hline C1S & 14 & p.K210T & c. $629 \mathrm{~A}>\mathrm{C}$ & rs782009231 & Mixed \\
\hline C2 & 15 & p.N310S & c. $929 \mathrm{~A}>\mathrm{G}$ & rs144201432 & All predict benign \\
\hline C2 & 16 & p.P724L & c. $2171 \mathrm{C}>\mathrm{T}$ & rs145050210 & All predict benign \\
\hline C3 & 17 & p.K155Q & c. $463 \mathrm{~A}>\mathrm{C}$ & rs147859257 & All predict benign \\
\hline C3AR1 & 18 & p.S308A & c. $922 \mathrm{~T}>\mathrm{G}$ & rs757235303 & All predict benign \\
\hline C8A & 19 & p.R485L & c.1454_1455delGCinsTT & rs386631447 & Mixed \\
\hline$C 8 A$ & 15 & p.D129N & c. $385 \mathrm{G}>\mathrm{A}$ & rs150404785 & Mixed \\
\hline C9 & 20 & p.D80N & c. $238 \mathrm{G}>\mathrm{A}$ & rs115196369 & All predict benign \\
\hline$C D 46$ & 21 & p.R82W & c. $244 \mathrm{C}>\mathrm{T}$ & rs200133631 & Mixed \\
\hline$C F B$ & 22 & p.I242L & c. $724 \mathrm{~A}>\mathrm{C}$ & rs144812066 & Mixed \\
\hline$C F B$ & 17 & p.S284N & c. $851 G>A$ & rs756948669 & All predict benign \\
\hline $\mathrm{CFH}$ & 17 & p.Q950H & c. $2850 \mathrm{G}>\mathrm{T}$ & rs149474608 & Mixed \\
\hline $\mathrm{CFH}$ & 23 & p.R166L & c. $497 \mathrm{G}>\mathrm{T}$ & None & Mixed \\
\hline CFH & 24 & Splice site & c. $3134-5 \mathrm{~T}>\mathrm{A}$ & rs513699 & Mixed \\
\hline CFH & 25 & p.P43T & c.127C > A & rs764941928 & All predict benign \\
\hline $\mathrm{CFH}$ & 26 & p.T956M & c. $2687 \mathrm{C}>\mathrm{T}$ & rs145975787 & All predict benign \\
\hline CFHR4 & 27 & p.Y362H & c. $1084 \mathrm{~T}>\mathrm{C}$ & rs201636212 & Mixed \\
\hline CFHR4 & 28 & p.W233C & c.699G >C & rs116119247 & Mixed \\
\hline CFHR4 & 29 & p.Y35H & c. $103 \mathrm{~T}>\mathrm{C}$ & rs138792300 & Mixed \\
\hline CFHR4 & 4 & p.L561I & c. $1681 \mathrm{C}>\mathrm{A}$ & rs80010185 & All predict benign \\
\hline CFHR5 & 30 & p.C568Ter & c. $1704 \mathrm{~T}>\mathrm{A}$ & rs143140599 & Mixed \\
\hline CFHR5 & 31 & p.G471E & c. $1412 \mathrm{G}>\mathrm{A}$ & rs144438200 & Mixed \\
\hline CFI & 32 & p.K267E & c.799 A > G & rs75612300 & Mixed \\
\hline$C F I$ & 33 & p.I416L & c. $1246 \mathrm{~A}>\mathrm{C}$ & rs61733901 & Mixed \\
\hline$C F I$ & 34 & p.H183R & c. $548 \mathrm{~A}>\mathrm{G}$ & rs75612300 & Mixed \\
\hline CR2 & 20 & p.V946M & c. $2836 \mathrm{G}>\mathrm{A}$ & rs147451324 & Mixed \\
\hline CR2 & 35 & p.R428H & c.1283G > A & rs183067414 & Mixed \\
\hline CR2 & 36 & Splice site & c. $2903-3 \mathrm{C}>\mathrm{A}$ & rs372214909 & All predict benign \\
\hline CR2 & 37 & p.S1016L & c. $3047 \mathrm{C}>\mathrm{T}$ & rs138062179 & All predict benign \\
\hline$M A S P 1$ & 2 & p.R288Q & c. $863 \mathrm{G}>\mathrm{A}$ & rs116001173 & Mixed \\
\hline$M A S P 1$ & 38 & p.R441H & c. $1322 \mathrm{G}>\mathrm{A}$ & rs141872329 & Mixed \\
\hline$P L G$ & 39 & p.T742N & c. $2225 \mathrm{C}>\mathrm{A}$ & None & Mixed \\
\hline$P L G$ & 40 & Splice site & c. $407+8 \mathrm{~A}>\mathrm{G}$ & None & All predict benign \\
\hline$P L G$ & 22 & p.D258N & c. $772 \mathrm{G}>\mathrm{A}$ & rs138242513 & All predict benign \\
\hline
\end{tabular}


COL4A3, COL4A4, COL4A5, COL4A6, COQ2, COQ4, COQ6, CRB2, DDX53, DGKE, DLC1, EMP2, FAT1, IL15RA, INF2, ITGA3, ITGB4, LAMB2, LMX1B, MAGI2, MYH9, MYO1E, NEIL1, NPHS1, NPHS2, NUP205, NUP93, NXF5, OCRL, PAX2, PDSS1, PDSS2, PLCE1,PODXL, PTPRO, SCARB2, SHROOM3, SMARCAL1, TNS2, TRPC6, TTC21B, VEGFA, WT1 and XPO5). Seven of the 63 African Americans (11\%) were found to be homozygous for APOL1 risk alleles. The lack of enrichment for patients homozygous for APOL1 risk alleles indicates that hypertension-associated microangiopathic changes are not within the morphologic spectrum of APOL1-associated nephropathy.

\section{Discussion}

There is mounting evidence that a subset of patients with thrombotic microangiopathy display aberrant activation of complement pathways in clinical settings not thought to be related to complement abnormalities. Included, among others, are malignancy, autoimmune disease, infection, drugs, pregnancy-related conditions, post-transplant thrombotic microangiopathy (including use of calcineurin inhibitors) and malignant hypertension. ${ }^{2}$ Many of these patients are unlikely to undergo work-up for genetic defects of complement because the etiology of their thrombotic microangiopathy is assumed to be due to the secondary disease.

The prevalence of thrombotic microangiopathy in the setting of malignant hypertension is variable. Estimates range from 27 to $56 \% .{ }^{15,16}$ Renal insufficiency or failure is common in malignant hypertension, occurring either as an acute complication or a pre-existing condition. ${ }^{17,18}$ The triad of severe or malignant hypertension, renal failure and thrombotic microangiopathy is less frequent, especially at initial presentation. The natural inclination is to blame the thrombotic microangiopathy on the hypertension. Instead, several studies suggest that the triad is driven in some patients by aberrant activation of complement and therefore represents atypical hemolytic uremic syndrome ${ }^{4-6}$ For instance, a recent case series details nine patients with 'hypertension-associated thrombotic microangiopathy', who underwent genetic sequencing of the most common complement genes associated with atypical hemolytic uremic syndrome. ${ }^{4}$ Similar to our study, all of the patients presented with severe hypertension, renal failure and thrombotic microangiopathy changes on kidney biopsy. Six of the nine patients had mutations of complement genes (although one was a $C F H$ variant of unknown significance), indicative of atypical hemolytic uremic syndrome. The findings, however, are at odds with ours.

We performed a next-generation sequencing panel sequencing a total of 29 complement and coagulation cascade genes in 100 patients also presenting with severe hypertension and renal thrombotic microangiopathy A. A key difference, however, between our study and the case series by Timmermans et $a l^{4}$ is the biopsy morphology. All of the patients in the previous study showed changes of active thrombotic microangiopathy, including microthrombosis, mesangiolysis, fibrinoid arteritis and mucoid intimal edema. The high mutation frequency in this cohort is not surprising, as patients with atypical hemolytic uremic syndrome tend to show a higher degree of histologic activity than severe or malignant hypertension without hemolytic uremic syndrome. ${ }^{16} \mathrm{We}$, on the other hand, explored similar genetic defects in a patient population where hypertension rather than atypical hemolytic uremic syndrome was the likely driver of thrombotic microangiopathy -that is, in patients whose biopsies showed activity limited to mucoid intimal edema of the arterial tree. The rationale for this approach is based on the premise that arterial intimal edema (a.k.a, mucoid intimal hyperplasia) in isolation and without accompanying glomerular fibrin thrombi is a histologic sine qua non of thrombotic microangiopathy in severe hypertension..$^{7,8,11,12}$ No pathogenic variants were subsequently identified in our cohort, which differs sharply from the estimated $60-70 \%$ frequency of similar mutational events in atypical hemolytic uremic syndrome. ${ }^{1}$ However, there were variants identified which were classified as variants of uncertain significance including 13 patients with variants of uncertain significance that were predicted to be deleterious by all three in-silico prediction methods utilized. Thus, although the diagnosis of atypical hemolytic uremic syndrome was largely negated in our patient population, it cannot be completely excluded. Interestingly, seven patients had variants in genes tightly correlated with the lectin pathway including the C2, FCN1, MASP1 and MSP2 genes (Table 1). These data suggest the need for additional studies investigating the role of the lectin pathway of complement in similar patient populations.

When a patient presents with severe hypertension and renal thrombotic microangiopathy, the assumption that the thrombotic microangiopathy is caused by hypertension and not atypical hemolytic uremic syndrome is predicated on Bayesian logic. ${ }^{19}$ Asif et $a l^{6}$ suggest that a prior history of hypertension, signs of hypertensive heart disease, relatively high platelet count and retinopathy favor severe hypertension over atypical hemolytic uremic syndrome. Van Laecke and Van Biesen ${ }^{19}$ recommend performing an early eye fundus examination in these patients and withholding complement and genetic testing in those with grade III/IV retinopathy and diastolic blood pressures exceeding $130 \mathrm{~mm} \mathrm{Hg}$. Timing the genetic work-up for atypical hemolytic uremic syndrome relies on the clinical acumen, intuition and experience, as well as the patient's circumstance. There are no consensus guidelines on how to test and treat these types of patients. 
Forestalling the decision to test and waiting for results could delay treatment with eculizumab and result in excessive and irreversible damage to the kidneys. The decision is made somewhat easier by laboratories that are now able to turnaround a basic gene panel within a few days rather than a few weeks. It should be emphasized, although, that a negative result does not rule out atypical hemolytic uremic syndrome, as at least $30 \%$ of cases are not associated with a known mutational event. Failure to achieve (1) a reasonable response to blood pressure control with antihypertensive drugs, (2) resolution of the thrombotic microangiopathy over days to weeks and (3) remission or stabilization of kidney function, raise concerns for atypical hemolytic uremic syndrome.

The combined data from this case series and Timmermans et al ${ }^{4}$ suggests that the kidney biopsy may be informative by distinguishing between low versus high probability of atypical hemolytic uremic syndrome in patients with severe hypertension. Glomerular involvement and identifiable fibrin thrombi on the biopsy increase the likelihood of a genetic complement abnormality linked to the etiology of the thrombotic microangiopathy. ${ }^{4,5}$ Furthermore, the biopsy is helpful in determining the extent of chronic and irreversible disease. The clinical algorithm of Laurence et $a l^{3}$ should be initiated early in the course to either rule out atypical hemolytic uremic syndrome or differentiate it from thrombotic thrombocytopenia purpura and Shiga toxin-induced hemolytic uremic syndrome. About $25 \%$ of patients with atypical hemolytic uremic syndrome have coexisting disorders, which appear to serve as triggers. ${ }^{20}$ Thus, secondary forms of thrombotic microangiopathy should also be considered-namely, autoimmune disease (especially systemic lupus erythematosus, antiphospholipid antibody syndrome and scleroderma), bacterial and viral infections, disseminated intravascular coagulation, pancreatitis, pregnancy-associated hemolysis elevated liver enzymes low platelets (HELLP) syndrome, drugs, systemic cancer, Evan's disease, coagulopathic disorders, among others.

In summary, our study represents the largest to date detailing genetic variants in complement and coagulation genes in patients presenting with the triad of severe hypertension, renal dysfunction and thrombotic microangiopathy. We focused on a particular histopathologic variant of thrombotic microangiopathy. The study is limited by the incomplete clinical data at the time of biopsy. All patients were known to have renal failure and malignant hypertension, as well as the classic histopathology of hypertension-associated thrombotic microangiopathy. Other clinical parameters such as thrombocytopenia, lactate dehydrogenase, presence of autoantibodies, etc., were not available for all patients. Regardless, none of the patients surveyed harbored genetic variants diagnostic of complement-mediated disease, although some did have genetic variants of uncertain significance. Our results inform recent data by suggesting that patients who present with severe or malignant hypertension and renal thrombotic microangiopathy may be at higher risk for atypical hemolytic uremic syndrome only if the biopsy shows more active disease that includes glomerular fibrin thrombi.

\section{Disclosure/conflict of interest}

This study was funded through an investigator initiated grant from Alexion Pharmaceuticals Inc., which has not had any influence on the results or interpretations in this article. The authors declare no conflict of interest.

\section{References}

1 Nester CM, Barbour T, de Cordoba SR, et al. Atypical aHUS: state of the art. Mol Immunol 2015;67:31-42.

2 George JN, Nester CM. Syndromes of thrombotic microangiopathy. N Engl J Med 2014;371:654-666.

3 Laurence J, Haller H, Mannuccio Mannucci $\mathrm{P}$, et al. Atypical hemolytic uremic syndrome (aHUS): essential aspects of an accurate diagnosis. Clin Adv Hematol Oncol 2016;14:1-16.

4 Timmermans SA, Abdul-Hamid MA, Vanderlocht J, et al. Patients with hypertension-associated thrombotic microangiopathy may present with complement abnormalities. Kidney Int 2017;91:1420-1425.

5 Totina A, Iorember F, El-Dahr SS, et al. Atypical hemolytic-uremic syndrome in a child presenting with malignant hypertension. Clin Pediatr (Phila) 2013;52: 183-186.

6 Asif A, Nayer A, Haas CS. Atypical hemolytic uremic syndrome in the setting of complement-amplifying conditions: case reports and a review of the evidence for treatment with eculizumab. J Nephrol 2017;30: 347-362.

7 Heptinstall RH. Renal biopsies in hypertension. $\mathrm{Br}$ Heart J 1954;16:133-141.

8 Churg J, Strauss L, Paronetto F. Some aspects of the pathology of hypertension. Vascular lesions in experimental and human hypertension. J Mt Sinai Hosp NY 1961;28:111-128.

9 Zhang B, Xing C, Yu X, et al. Renal thrombotic microangiopathies induced by severe hypertension. Hypertens Res 2008;31:479-483.

10 Shavit L, Reinus C, Slotki I. Severe renal failure and microangiopathic hemolysis induced by malignant hypertension-case series and review of literature. Clin Nephrol 2010;73:147-152.

11 Jennette CJ, D'Agati V, Olson JL, et al. Heptinstall's Pathology of the Kidney7 ed.Lippincott Williams and Wilkins: Philadelphia, PA, USA, 2014, p 759.

12 Colvin RB, Chang AC. Diagnostic Pathology: Kidney Diseases2 ed.Amirsys: Philadelphia, PA, USA, 2015, p 523.

13 Larsen CP, Durfee T, Wilson JD, et al. A custom targeted next-generation sequencing gene panel for the diagnosis of genetic nephropathies. Am J Kidney Dis 2016;67:992-993. 
14 Richards S, Aziz N, Bale S, et al. Standards and guidelines for the interpretation of sequence variants: a joint consensus recommendation of the American College of Medical Genetics and Genomics and the Association for Molecular Pathology. Genet Med 2015;17:405-424.

15 van der Born BJ, Honnebier UP, Koopmans RP, et al. Microangiopathic hemolysis and renal failure in malignant hypertension. Hypertension 2005;45:246-251.

$16 \mathrm{Yu}$ XJ, Yu F, Song D, et al. Clinical and renal biopsy findings predicting outcome in renal thrombotic microangiopathy: a large cohort study from a single institute in China. Sci World J 2014;2014:680502.

17 Amraoui F, Bos S, Vogt L, et al. Long-term renal outcome in patients with malignant hypertension: a retrospective cohort study. BMC Nephrol 2012;13:71.

18 van den Born BJ, Koopmans RP, Groeneveld JO, et al. Ethnic disparities in the incidence, presentation and complications of malignant hypertension. J Hypertens 2006;24:2299-2304.

19 Van Laecke S, Van Biesen W. Severe hypertension with renal thrombotic microangiopathy: what happened to the usual suspect? Kidney Int 2017;91: 1271-1274.

20 Noris M, Caprioli J, Bresin E, et al. Relative role of genetic complement abnormalities in sporadic and familial aHUS and their impact on clinical phenotype. Clin J Am Soc Nephrol 2010;5:1844-1859.

This work is licensed under a Creative Commons Attribution-NonCommercialNoDerivs 4.0 International License. The images or other third party material in this article are included in the article's Creative Commons license, unless indicated otherwise in the credit line; if the material is not included under the Creative Commons license, users will need to obtain permission from the license holder to reproduce the material. To view a copy of this license, visit http:/creativecommons.org/licenses/ by-nc-nd/4.0/

(C) The Author(s) 2018 